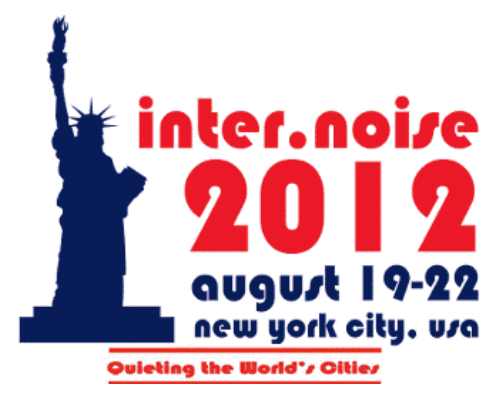

\title{
Overview of an indoor sonic boom simulator at NASA Langley Research Center
}

\author{
Jacob Klos ${ }^{1)}$ \\ Structural Acoustics Branch \\ NASA Langley Research Center \\ Hampton, VA 23681
}

A facility has been constructed at NASA Langley Research Center to simulate the soundscape inside residential houses that are exposed to environmental noise from aircraft. This controllable indoor listening environment, the Interior Effects Room, enables systematic study of parameters that affect psychoacoustic response. The single-room facility, built using typical residential construction methods and materials, is surrounded on adjacent sides by two arrays of loudspeakers in close proximity to the exterior walls. The arrays, containing 52 subwoofers and 52 mid-range speakers, have a usable bandwidth of $3 \mathrm{~Hz}$ to $5 \mathrm{kHz}$ and sufficient output to allow study of sonic boom noise. In addition to these exterior arrays, satellite speakers placed inside the room are used to augment the transmitted sound with rattle and other audible contact-induced noise that can result from low frequency excitation of a residential house. The layout of the facility, operational characteristics, acoustic characteristics and equalization approaches are summarized.

\section{INTRODUCTION}

Supersonic flight over land is currently prohibited by the FAA and other international regulatory bodies due to the effects of sonic boom noise on populations over which the aircraft would fly. These restrictions were put in place based on research performed in the 1960's, which studied environmental noise and community annoyance resulting from supersonic aircraft designs of that era. While at cruise, these designs produce sonic boom signatures on the ground with a classic $\mathrm{N}$-wave shape and a peak overpressure typically in the range of 1 to $2 \mathrm{lb}_{\mathrm{f}} / \mathrm{ft}^{2}$. This en-route noise was determined to be too intrusive to communities. However, there is currently a desire among airplane manufacturers to build aircraft capable of flying overland at supersonic speeds. This desire is motivated in part by the demonstration of sonic boom shaping by DARPA, NASA, and industrial partners during a recent experiment ${ }^{1}$. In that experiment, it was shown the character of a sonic boom could be manipulated in a predictable way by shaping the airframe. By tailoring the fuselage shape, future commercial supersonic aircraft at cruise will produce a sonic boom on the ground that is significantly quieter than previous generation aircraft.

\footnotetext{
1) j.klos@nasa.gov
} 
Unrestricted supersonic flight over land is required for such a vehicle to be economically viable. Thus, a rule change is required to replace the current prohibition with a noise based criterion. Fundamental research into the intrusiveness of these low amplitude booms is needed, and people's reaction to noise experienced inside buildings is a primary concern ${ }^{2,3}$. The facility described here allows systematic and independent simulation of the noise sources that would be present inside a house exposed to a sonic boom. Current research using the facility is focused on identifying a psychoacoustic model that describes people's reaction to this environmental noise source. This model will help inform the regulatory process.

\section{FACILITY DESCRIPTION}

A plan view of the Interior Effects Room (IER) is shown in Fig. 1. The interior dimensions of the furnished test space where subjects are seated during a test (Fig. 2) are 11'-4" by 13'-5" with an 8'-4" high ceiling. Walls consist of wooden $2 \times 4$ stud construction, nominally 16 " on center, with a $1 / 2$ " plywood exterior sheathing and 1/2" gypsum board interior finish (Fig. 3). Exterior finishing materials typical of residential construction, such as stucco or siding, were not used on the exterior façade. The facility, whose floor is framed with $2 \times 10$ " wood joists nominally 16 " on center, is built atop a raised platform that provides a 21" high crawl space. This enables placement of shakers under the facility floor to generate tactile input that can be controlled separately from the audible content generated by the speaker systems shown in Figs. 4 to 6 . The subfloor is $3 / 4$ " plywood, and is finished with a floating laminate flooring system. Carpeting can be added to or removed from the room to affect the acoustical absorption. The room includes two closets (Fig. 1) that have louvered doors, inside of which 3' deep melamine foam wedges can be placed (Fig. 7) to further alter the acoustical absorption of the room.

Two large, movable speaker arrays are located outside of the test space adjacent to two walls enclosing the test space (Figs. 1, 4 and 5). The array adjacent to the shorter wall (array \#1) consists of 24 subwoofer and mid-range speaker pairs, 6 pairs along the length of the wall by 4 pairs high. The array exterior to the larger wall, which contains a double hung window, consists of 28 pairs of speakers (array \#2). A cross section of an exterior array assembly in relation to the interior test space is shown in Fig. 4. These speaker arrays are hung from the concrete ceiling of the room in which the IER is built so the weight of the speaker arrays is not borne by the wooden structure of the IER. Each movable speaker array mates to a wooden flange framed with $4 \times 4$ timbers that are permanently affixed to the perimeter of each adjacent wall. This flange forms a 14" deep enclosed air cavity in-between the exterior sheathing of the wall and the array of subwoofers when the arrays are mated to the flange. To reduce the influence of acoustic modes that would form within this air cavity, a 3/4" fiberboard framework lined with 3" thick melamine foam sheets is attached to the flange (Fig. 5a). This fiberboard framework forms baffled cells within the air cavity, which are approximately 22"x22"x14" in volume, where each subwoofer and mid-range pair drives one cell (Fig. 5b). Acoustically resistive elements are installed in the speaker array (Fig. 5c, red arrow) to vent the air cavity that is formed when the speaker array is mated to the flange. This resistive leak reduces the influence of the acoustical stiffness, formed by the baffled air cavity, on the IER wall vibration. These two exterior arrays are used to create a desired waveform that excites the exterior façade of the IER, enabling simulations of aircraft environmental noise impinging on and transmitting through the walls of a typical residence.

In addition to the exterior arrays, seven satellite speakers and a subwoofer are located inside the test space. These interior speakers are used to play back either recordings or simulations of rattle and other contact induced noise sources that typically result when low frequency sound excites a residential house. Five of the seven satellites are highlighted in Fig. 6 (orange arrows). 
Two speakers are mounted adjacent to the window to simulate rattle noise radiated by the window. One speaker is mounted above the ceiling fan. Two additional movable satellites can be placed on shelves in the room to simulate sounds from bric-a-brac. Two speakers are mounted at ceiling-height, only one of which is pictured. The satellite speaker that is not pictured in Fig. 6 is located above a closet door (Fig. 7, orange arrow). These ceiling-height speakers can be used to play back wall creak and door rattle. The interior satellites and subwoofer have a usable bandwidth of approximately $80 \mathrm{~Hz}$ to $20 \mathrm{kHz}$.

Each cell of the two exterior speaker arrays and each interior satellite speaker are independently controlled by a separate 24-bit digital to analog converter (D/A). Each D/A channel is routed to a analog-to-digital-to-analog audio processor that acts as a crossover, splitting the full bandwidth analog signal into separate low and high frequency signals that are amplified and sent to each speaker. This arrangement allows for independent control of the 112 speakers within the IER (52 exterior subwoofers, 52 exterior mid-range, 7 interior satellites, and 1 interior subwoofer). The crossover filters and audio amplifiers used in the IER are pro-audio grade hardware typically used in sound reinforcement applications. As such, both components have $3 \mathrm{~Hz}$ AC coupling filters that introduce signal attenuation and delay below this cutoff frequency. Compensation for this and other coloration in the audio system and the facility capabilities are discussed in the next section.

\section{ACOUSTICAL CHARACTERISTICS}

\subsection{Equalization of the Exterior Arrays}

A temporally and spectrally accurate simulation of a desired exterior stimulus is required. Thus, the audio system should, ideally, have a frequency response with unity gain over the bandwidth required to reproduce the desired aircraft noise source. In practice, the frequency response magnitude and phase will not be flat and equalization will be required to compensate for coloration from the:

- Electro-mechanical characteristics of the speakers

- Frequency dependent radiation from the speakers

- Acoustical response of the baffled air cavity

- Low frequency roll-off of the AC coupling filters in the crossovers and amplifiers

To perform a system identification and equalize the exterior arrays, microphones were centered inside each of the baffled cells, flush against the exterior of the facility wall (Fig. 5b, blue arrow). These are used to compute the frequency response between the D/A output voltage and the exterior pressure. The magnitude of the un-equalized frequency response function (FRF) of array \#1, averaged across the cells in that array, is shown in Fig. 8 (blue line).

To reproduce sonic boom noise the FRF must be flat from about $1 \mathrm{~Hz}$ to $5 \mathrm{kHz}$. A two-step equalization approach is used to remove the coloration of the audio system within this frequency range. First, parametric equalization is implemented in the crossovers to decrease the dynamic range of the FRF. The result of incorporating parametric equalization into the audio chain is illustrated in Fig. 8 (red line). The FRF dynamic range is reduced from $60 \mathrm{~dB}$ (blue line) to about $18 \mathrm{~dB}$ (red line). This reduction significantly improves the result that is obtained from the second equalization step. A Wiener filtering approach ${ }^{4}$ is used to create a finite impulse response (FIR) filter to correct the remaining coloration and the offset in gain. The resulting FIR filter, the frequency response of which is shown in Fig. 9, is used to pre-distort a desired waveform prior to playback through the audio system. The frequency response of the equalized audio system incorporating both parametric equalization and this FIR filter is shown in Fig. 8 (green line). 
Nearly unity gain with a reasonably flat phase response is obtained using the combination of parametric equalization and pre-distortion of the desired waveform.

\subsection{Equalization of the Interior Satellites}

The interior satellite speakers and subwoofer are used to simulate rattle from structural elements or from bric-a-brac placed inside the room that typically occurs when a building is by a low-frequency noise source. Efforts were made to minimize naturally occurring rattles so rattle levels can be completely controlled using these satellite speakers. Compensation must be made for the coloration added by the dynamics of the speakers and room effects. However, a Wiener filter approach is impractical in this instance due to the abundance of room modes inside the IER. Instead, a combination of parametric equalization and one-third-octave band graphic equalization is used. Since both approaches are implemented in the audio hardware, no predistortion of the desired rattle waveform is needed prior to playback. The one-third-octave band response of a typical satellite and the subwoofer, averaged across eight randomly selected receiver locations inside the room, is shown in Fig. 10 (red line). The mean response within the room is flat to within approximately $\pm 2 \mathrm{~dB}$ from $100 \mathrm{~Hz}$ to $10 \mathrm{kHz}$. However, a large variation in the one-third-octave spectra is observed across the eight measurement locations within the room (Fig. 10, blue lines). The variation in sound levels among locations within the room should be considered when designing a test utilizing these speakers.

\subsection{Performance of the Exterior Arrays}

A synthetic sonic boom of 32-Pa overpressure and 0.170-sec duration was pre-distorted, see section 3.1 for details, and played back through array \#2. The sound pressure measured at the exterior surface of the wall, averaged across the baffled cells, is shown in Fig. 11 and is compared to the desired signal. Small deviation from the desired waveform is observable in the measured waveform, which is likely attributed to small deviations about a unity gain in the equalized frequency response (Fig. 8, green line). However, a visual comparison of the waveform reproduced by the facility to the desired stimulus is misleading. Visual inspection does not appropriately emphasize audible features present in the reproduced sound. To quantify the accuracy of the exterior simulation, seventy three sonic booms having a range of amplitude, rise time, and duration that were measured on the ground during field experiments ${ }^{5}$ were predistorted and played back through both exterior arrays. Perceived Level (PL) ${ }^{6}$, which is a loudness metric that correlates well with people's reaction to sonic boom noise ${ }^{7,8}$, was computed for each of the 73 pairs of measured and desired waveforms. The error between the Perceived Level of the measured and desired waveform was found for each pair and is plotted against the loudness of the desired stimuli in Fig. 12. The error ranges from approximately -0.7 to $+0.6 \mathrm{PL}$ for sonic boom stimuli that range in loudness from 70 to 105 PL. Other loudness metrics have a similar range in error.

The maximum sound pressure level that can be produced by the exterior arrays is limited by the subwoofer capabilities. The allowable displacement of the subwoofer diaphragm limits the exterior sound level below the mechanical resonance frequency of the speaker and the power delivered to the voice coil limits the level at higher frequencies. The frequency dependent maximum sound pressure level and the resulting operational envelope of the exterior arrays is shown in Fig. 13. The envelope identifies the maximum sound pressure level that can be obtained at the exterior surface of the IER walls when the arrays are driven by a pure tone at a particular frequency on the abscissa. This should not be confused with the frequency response of 
an array, which relates the voltage output by the D/A converter to the sound pressure produced by the array (Fig. 8, green line).

Since a sonic boom is a low frequency transient, and the curves in Fig. 13 were defined using sinusoidal excitation, the maximum sonic boom output the arrays can attain is difficult to quantify precisely. It is mainly dependent on the duration of the sonic boom. In general, N-waves can be faithfully reproduced by the arrays if the overpressure is $30 \mathrm{~Pa}$ or less and the duration is 0.17 sec or less (Fig. 11). Above this level or duration, displacement of the subwoofer diaphragm is a limiting factor due to the roll-off of the arrays below $3 \mathrm{~Hz}$ (Fig. 13). Thus, it is recommended that desired signals be high pass filtered using a second order Butterworth filter with a $6 \mathrm{~Hz}$ corner frequency. Using this filtering, booms with an overpressure of at least $125 \mathrm{~Pa}$ can be reproduced. Also, this filtering has a negligible effect on the loudness of the stimulus and should not affect people's response to these sounds.

\subsection{Transmission Characteristics of the Facility}

Stimuli generated by the exterior arrays are attenuated by the walls of the facility as they transmit into the test space. This simulates the noise reduction that would occur in a typical house that is exposed to environmental noise. To quantify the noise reduction, pink noise was played through both exterior arrays. The noise reduction was defined as the ratio of the exterior pressure to the interior pressure averaged across nine randomly selected receiver locations. The one-third-octave band noise reduction for four different room configurations (Table 1) is listed in Table 2. In addition, the reverberation time was measured inside the IER for each room configuration and is listed in Table 3. A range of reverberation times is observed, which may affect the character of rattle sounds produced using the interior satellite speakers.

In order to determine if the transmission characteristics of the facility are similar to a residential structure exposed to real sonic booms, comparisons were made between indoor signatures measured in the test space and those obtained in an instrumented house exposed to sonic booms from an F-18 aircraft ${ }^{5}$. Seventy three sonic booms of varying Perceived Level were played through the exterior arrays. For each of these stimuli, sound pressure measurements were made inside the test space at nine randomly selected receiver locations and the loudness level was computed at each location. The location-averaged relationship between interior and exterior Perceived Level for two different array configurations is shown in Fig. 14 (red lines). The upper red curve is the case when booms are played through both arrays and the bottom red curve is the case when only array \#1 is used. As expected, the level inside the facility increases when both arrays are used. These data from the IER are compared to eight different receiver locations inside the house ${ }^{5}$. Five of those locations are in two different rooms on the shadow side of the house (black lines) and three receiver locations are in two rooms on the incident side of the house (blue lines). There is reasonably good agreement between the levels in the IER and those observed in the house exposed to sonic booms from an F-18 aircraft.

Ideally, subjects seated inside the test space would be exposed to the same transmitted sound. However, this will not be the case due to spatial variation caused by the low frequency modal response of the room and varying distance from the excited walls. For each of 73 sonic boom stimuli played through the exterior arrays, the loudness at the nine interior receiver locations and the best-fit line are shown in the top row of Fig. 15. Two different array configurations are included in the columns of Fig. 15. The residuals are shown in the middle row (Fig. 15), which give insight on the spatial variations that can be expected inside the facility. The distribution of the residuals and the standard deviation is shown in the bottom row (Fig. 15). The spatial variation of Perceived Level inside the test space is largest when only one array is driven 
and the $95 \%$ confidence interval is typically \pm 4 PL or smaller. Care should be taken when designing a psychoacoustic test to account for the effects of spatially varying acoustic levels.

\section{FACILITY OPERATION}

The audio hardware, signal playback and test flow are controlled by Labview software. Test stimuli, stored as .wav files, are progressively played via a playlist file. The stimulus sequence, inter-stimulus delay, inter-stimulus pauses, stimulus amplitude, and the judgments subjects are asked to make are defined within the playlist file. During subjective tests, questions are presented to subjects using images (Fig. 16) displayed remotely on laptop or tablet computers that are given to each subject seated in the room (Fig. 2). Subjects register a judgment by either marking a line scale, clicking an option within a form, or entering numeric input. To date, the software has required the definition of stimulus sequence and amplitude in advance. For future tests, the software will be updated to allow real time variation of stimulus amplitude and sequence, which will enable more flexibility in the types of tests that can be conducted.

\section{SUMMARY}

A new facility has been built at NASA Langley Research Center to simulate the soundscape inside residential houses exposed to sonic boom noise from aircraft. The physical, operational and acoustical characteristics have been reviewed in this paper. The unique capability afforded by this facility will be used to study people's reaction to sonic boom noise that transmits indoors. A psychoacoustic model is sought that describes people's response to this source of environmental noise. Ultimately, the metrics identified using this facility will help define a noise based criterion to replace the current regulation prohibiting overland supersonic flight.

\section{REFERENCES}

1. K.J. Plotkin, J.A. Page, D.H. Graham, J.W. Pawlowski, D.B. Schein, P.G. Coen, D.A. McCurdy, E.A. Haering, J.E. Murray, L.J. Ehernberger, D.J. Maglieri, P.J. Bobbitt, A. Pilon, J. Salamone, "Ground Measurements of a Shaped Sonic Boom," Proceedings of the 10th AIAA/CEAS Aeroacoustics Conference, Paper number AIAA 2004-2923, 2004.

2. C. W. Nixon, P. N. Borsky, "Effects of sonic boom on people: St. Louis, Missouri, 19611962,” The Journal of the Acoustical Society of America, vol. 39, no. 5B, 1966.

3. J. M. Fields, "Reactions of residents to long-term sonic boom noise environments," NASA Technical Report CR-201704, 1997.

4. S. Haykin, Adaptive Filter Theory, Prentice Hall, 1996.

5. J. Klos, 2008, "Vibro-Acoustic Response of Buildings Due to Sonic Boom Exposure: July 2007 Field Test,” NASA Technical Report TM-2008-215349, 2008.

6. K. P. Shepherd, B. M. Sullivan, "A loudness calculation procedure applied to shaped sonic booms,” NASA Technical Report TP-3134, 1993.

7. J. D. Leatherwood, B. M. Sullivan, "Loudness and annoyance response to simulated outdoor and indoor sonic booms,” NASA Technical Report TM-107756, 1993.

8. J. Rathsam, A. Loubeau, J. Klos, "A Study in a New Test Facility on Indoor Annoyance Caused by Sonic Booms," NASA Technical Report TM-2012-217332, 2012. 
Table 1 - Four of the ten room configurations tested during acoustic characterization of the facility.

\begin{tabular}{|c|cccccc|}
\hline $\begin{array}{c}\text { Room } \\
\text { Configuration }\end{array}$ & $\begin{array}{c}\text { Couch Inside } \\
\text { Room }\end{array}$ & $\begin{array}{c}\text { Chair Inside } \\
\text { Room }\end{array}$ & Rug Inside Room & $\begin{array}{c}\text { Foam Inside } \\
\text { Large Closet }\end{array}$ & $\begin{array}{c}\text { Foam Inside } \\
\text { Small Closet }\end{array}$ & $\begin{array}{c}\text { Closet Doors } \\
\text { Removed }\end{array}$ \\
\hline A & Yes & Yes & Yes & Yes & Yes & No \\
\hline E & Yes & Yes & Yes & No & No & No \\
\hline F & Yes & Yes & No & No & No & No \\
\hline H & No & No & No & No & No & Yes \\
\hline
\end{tabular}

Table 2 - Exterior-to-interior noise reduction, in $d B$, of the IER for four different room configurations when driving both exterior arrays with pink noise.

\begin{tabular}{|c|c|c|c|c|c|c|c|c|c|}
\hline \multirow{2}{*}{$\begin{array}{c}\text { Center } \\
\text { Frequency, } \mathrm{Hz}\end{array}$} & \multicolumn{4}{|c|}{ Room Configuration } & \multirow{2}{*}{$\begin{array}{c}\text { Center } \\
\text { Frequency, } \mathrm{Hz}\end{array}$} & \multicolumn{4}{|c|}{ Room Configuration } \\
\hline & A & $E$ & $\mathrm{~F}$ & $\mathrm{H}$ & & A & $E$ & $\mathrm{~F}$ & $\mathrm{H}$ \\
\hline 6.3 & 23.4 & 23.0 & 23.1 & 23.1 & 200 & 22.3 & 21.8 & 22.1 & 21.4 \\
\hline 8 & 22.6 & 22.2 & 22.2 & 22.3 & 250 & 19.8 & 19.3 & 19.3 & 18.0 \\
\hline 10 & 21.1 & 20.6 & 20.7 & 20.7 & 315 & 20.2 & 19.8 & 19.9 & 18.7 \\
\hline 12.5 & 19.1 & 18.8 & 18.9 & 18.9 & 400 & 22.6 & 22.0 & 22.0 & 20.2 \\
\hline 16 & 24.2 & 23.5 & 23.7 & 23.6 & 500 & 27.3 & 26.6 & 26.4 & 25.0 \\
\hline 20 & 16.4 & 17.4 & 17.4 & 18.3 & 630 & 34.8 & 34.2 & 34.1 & 32.8 \\
\hline 25 & 8.6 & 9.3 & 9.4 & 10.1 & 800 & 34.1 & 33.4 & 33.2 & 31.8 \\
\hline 31.5 & 9.7 & 9.6 & 9.6 & 10.6 & 1000 & 37.0 & 36.7 & 36.0 & 33.8 \\
\hline 40 & 12.6 & 12.0 & 12.1 & 11.2 & 1250 & 40.9 & 40.4 & 39.7 & 37.4 \\
\hline 50 & 19.6 & 20.3 & 20.4 & 20.3 & 1600 & 41.7 & 41.1 & 40.6 & 38.0 \\
\hline 63 & 19.6 & 19.7 & 19.9 & 20.8 & 2000 & 43.1 & 42.6 & 42.0 & 39.4 \\
\hline 80 & 18.0 & 15.8 & 15.9 & 15.6 & 2500 & 47.8 & 47.4 & 46.5 & 44.3 \\
\hline 100 & 21.6 & 21.6 & 21.7 & 22.0 & 3150 & 50.9 & 50.6 & 50.0 & 47.6 \\
\hline 125 & 18.9 & 17.5 & 18.0 & 17.8 & 4000 & 56.5 & 55.9 & 55.3 & 52.8 \\
\hline 160 & 15.8 & 17.0 & 17.0 & 15.3 & 5000 & 60.8 & 60.6 & 59.7 & 57.5 \\
\hline
\end{tabular}

Table 3 - Reverberation time estimates, T60 in seconds, for four different room configurations.

\begin{tabular}{|c|cccc|c|cccc|}
\hline Center & \multicolumn{4}{|c|}{ Room configuration } & \multicolumn{4}{c|}{ Center } & \multicolumn{4}{c|}{ Room configuration } \\
Frequency, $\mathrm{Hz}$ & $\mathrm{A}$ & $\mathrm{E}$ & $\mathrm{F}$ & $\mathrm{H}$ & Frequency, $\mathrm{Hz}$ & $\mathrm{A}$ & $\mathrm{E}$ & $\mathrm{F}$ & $\mathrm{H}$ \\
\hline $40^{*}$ & 0.56 & 0.77 & 0.80 & 0.76 & 1000 & 0.41 & 0.53 & 0.60 & 1.05 \\
\hline $50^{*}$ & 0.38 & 0.49 & 0.47 & 0.50 & 1250 & 0.42 & 0.52 & 0.61 & 1.14 \\
\hline $63^{*}$ & 0.48 & 0.57 & 0.55 & 0.42 & 1600 & 0.43 & 0.54 & 0.61 & 1.18 \\
\hline $80^{*}$ & 0.36 & 0.58 & 0.59 & 0.60 & 2000 & 0.39 & 0.47 & 0.56 & 1.14 \\
\hline $100^{*}$ & 0.27 & 0.50 & 0.50 & 0.54 & 2500 & 0.35 & 0.43 & 0.50 & 0.99 \\
\hline $125^{*}$ & 0.34 & 0.43 & 0.43 & 0.47 & 3150 & 0.35 & 0.41 & 0.49 & 0.93 \\
\hline $160^{*}$ & 0.26 & 0.27 & 0.25 & 0.39 & 4000 & 0.37 & 0.41 & 0.50 & 0.97 \\
\hline 200 & 0.27 & 0.35 & 0.34 & 0.42 & 5000 & 0.35 & 0.40 & 0.48 & 0.94 \\
\hline 250 & 0.39 & 0.46 & 0.45 & 0.64 & 6300 & 0.34 & 0.37 & 0.45 & 0.86 \\
\hline 315 & 0.41 & 0.53 & 0.51 & 0.72 & 8000 & 0.32 & 0.34 & 0.41 & 0.75 \\
\hline 400 & 0.42 & 0.50 & 0.53 & 0.82 & 10000 & 0.29 & 0.32 & 0.38 & 0.64 \\
\hline 500 & 0.43 & 0.51 & 0.58 & 0.88 & 12500 & 0.26 & 0.28 & 0.32 & 0.52 \\
\hline 630 & 0.41 & 0.54 & 0.58 & 0.88 & 16000 & 0.24 & 0.25 & 0.28 & 0.44 \\
\hline 800 & 0.41 & 0.54 & 0.58 & 0.95 & & & & & \\
\hline
\end{tabular}

* indicates frequencies below the Schroeder frequency of the room (approximately $190 \mathrm{~Hz}$ ) 


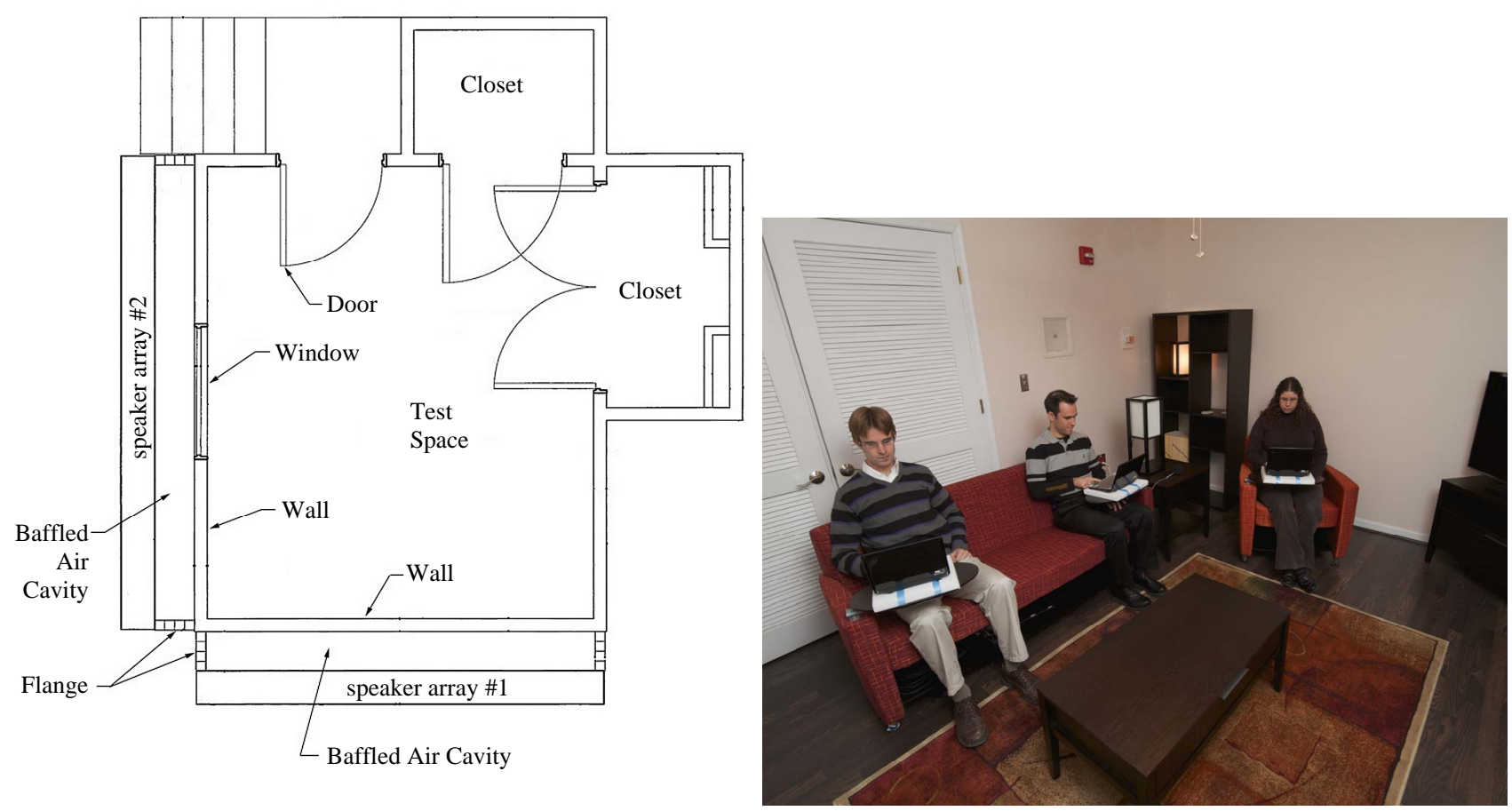

Fig. 1 - Plan view of the facility.

Fig. 2 - Subjects seated in the furnished test space.

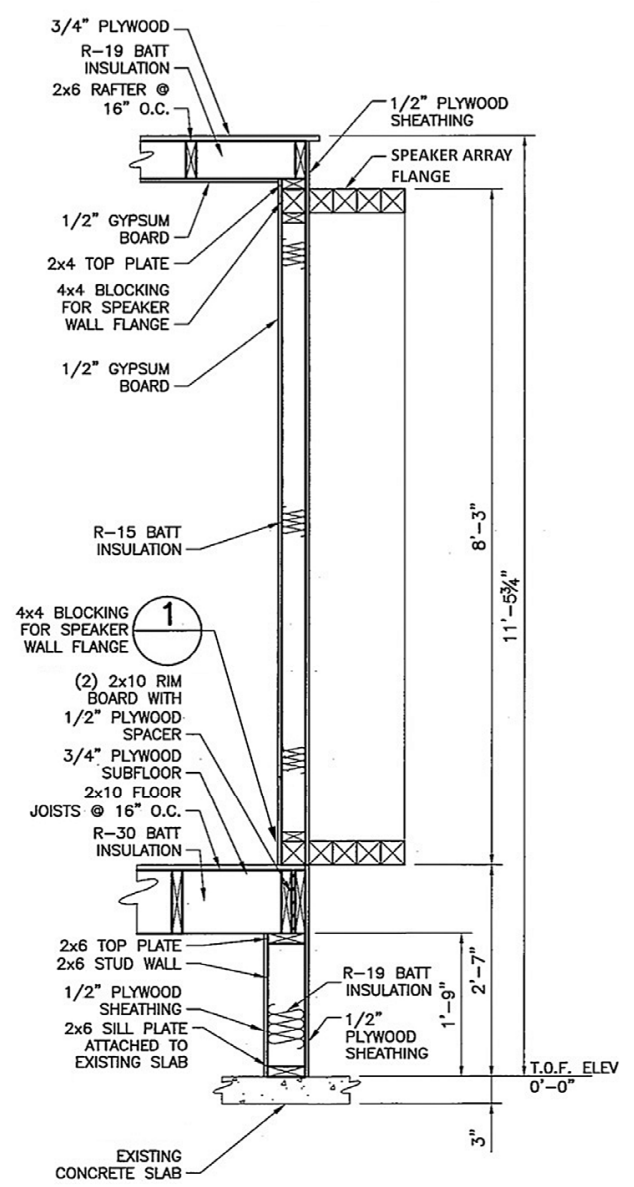

Fig. 3 - Typical wall cross section.

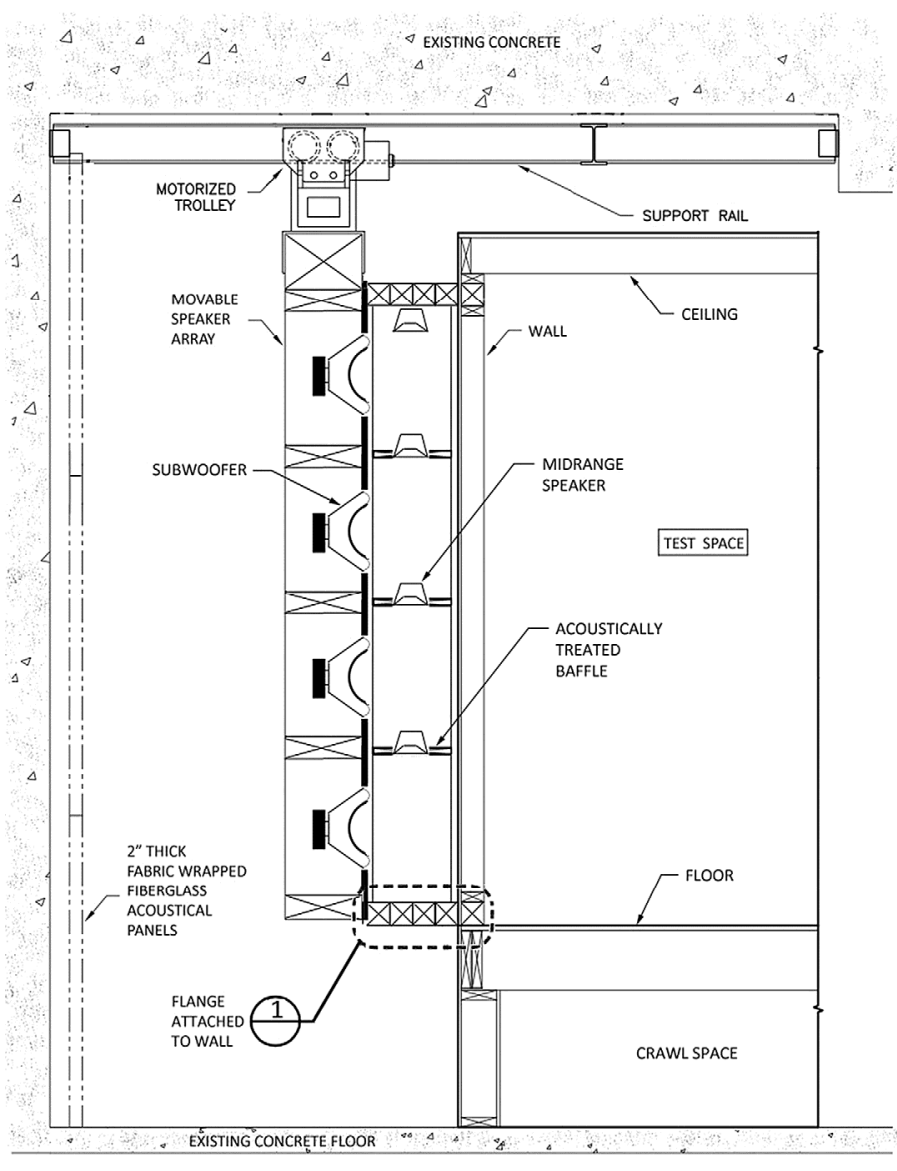

Fig. 4 - Cross section of the exterior speaker array. 


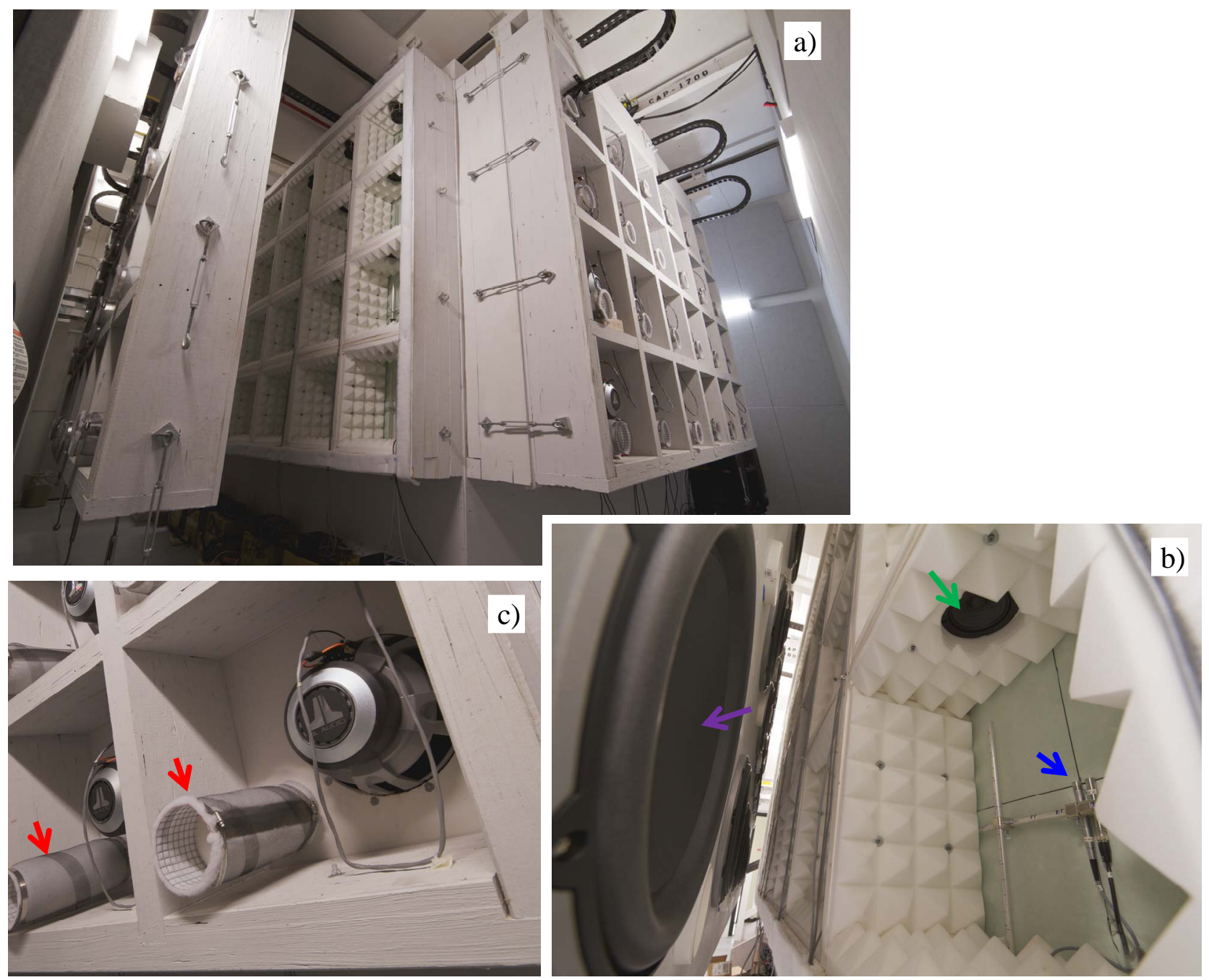

Fig. 5 - Exterior of the facility; a) the two speaker arrays with one array retracted showing the baffled cavity, b) one cell in the baffle showing a microphone $\rightarrow$, a mid-range $\rightarrow$, and a subwoofer speaker $\rightarrow$, and c) resistive elements $\rightarrow$ used to vent each baffled air cavity cell.

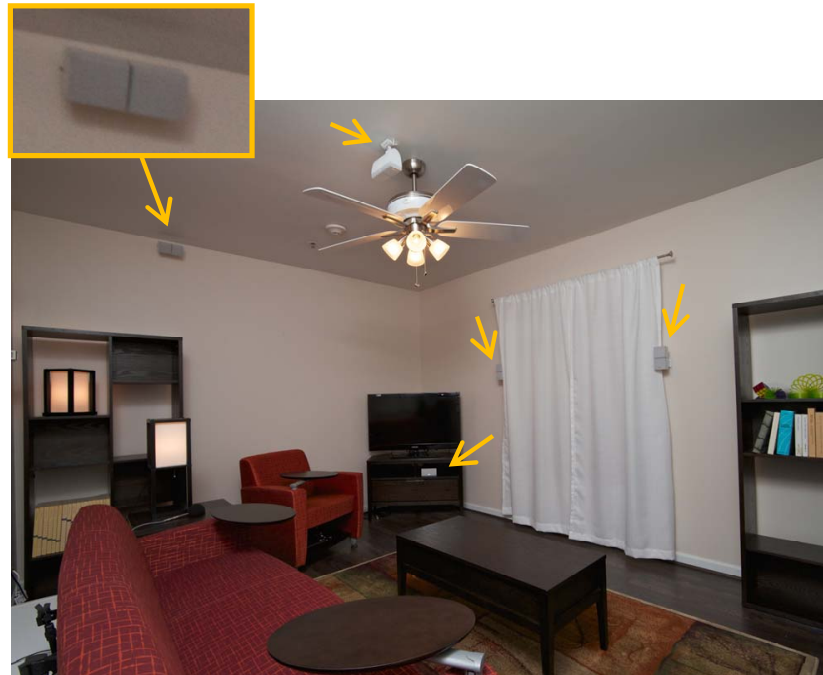

Fig. 6 - Interior of the facility, $\rightarrow$ five of seven satellite speakers used to play rattle stimuli.

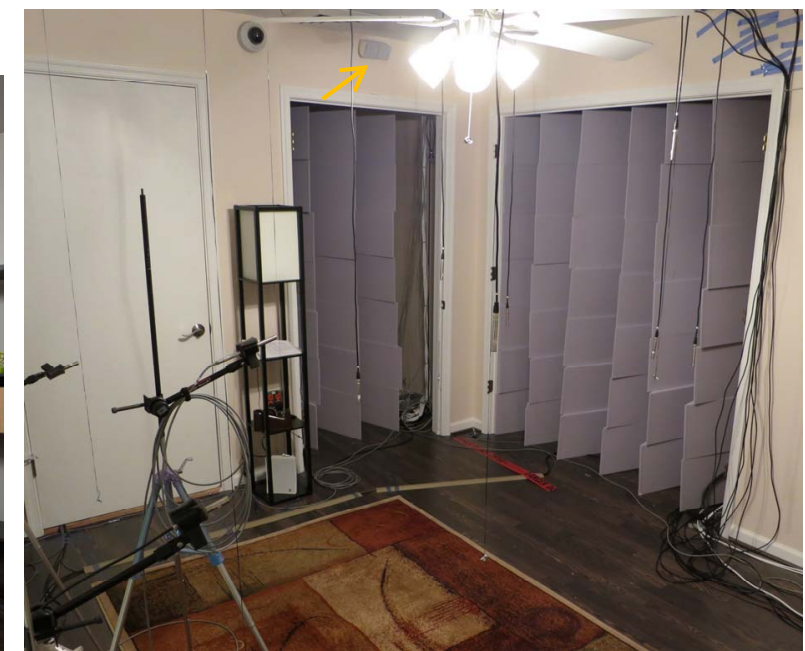

Fig. 7 - Closets filled with foam wedges. 

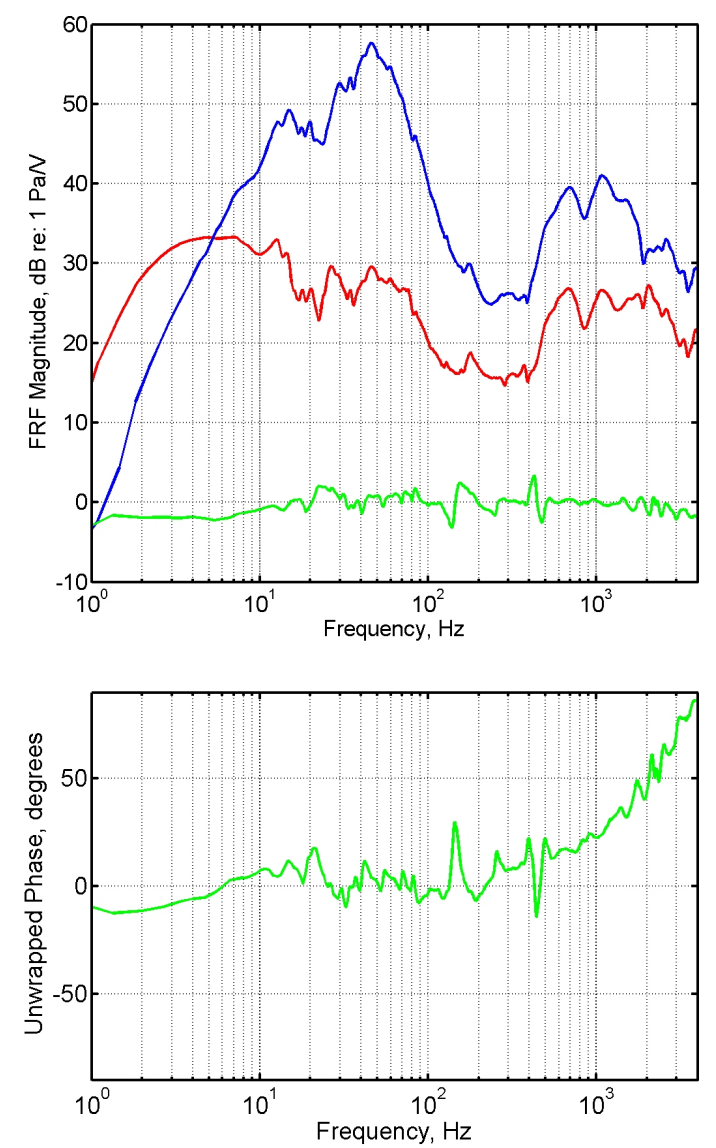

Fig. 8 - Comparison of the frequency response of array \#1; "-" without equalization, "-" with parametric equalization implemented in the crossovers, and "-" fully equalized.

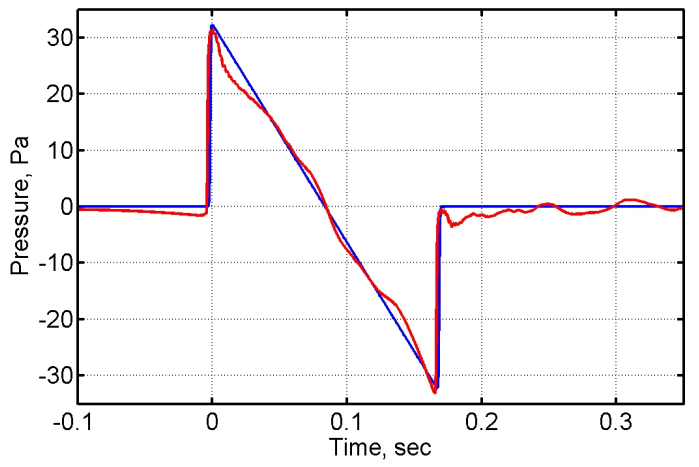

Fig. 11 - A ideal N-wave; "-" measured at the exterior of array \#2 and"-" the desired waveform.
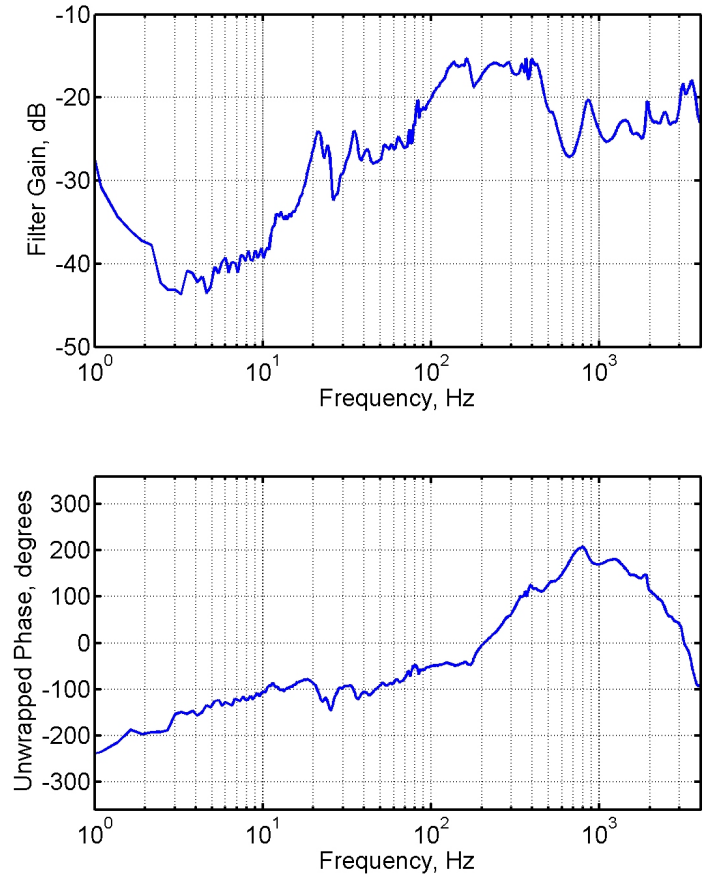

Fig. 9 - Frequency response of the FIR equalizer filter used to correct for the coloration of array \#1.

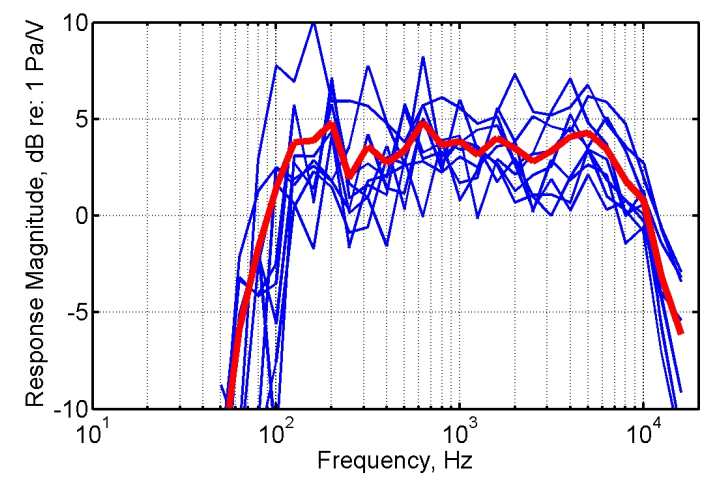

Fig. 10 - Response of a typical interior satellite speaker; at "-" 8 random locations inside the IER and"-" the mean response.

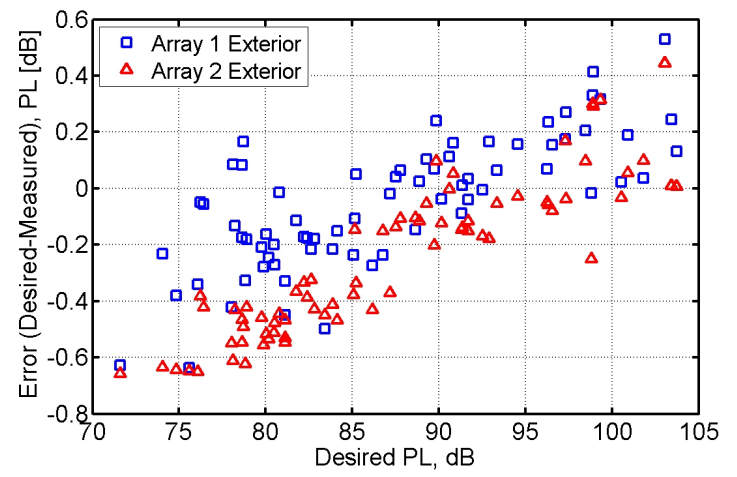

Fig. 12 - Error in the measured loudness, Perceived Level, at the exterior of the IER for playback of 73 different sonic booms. 


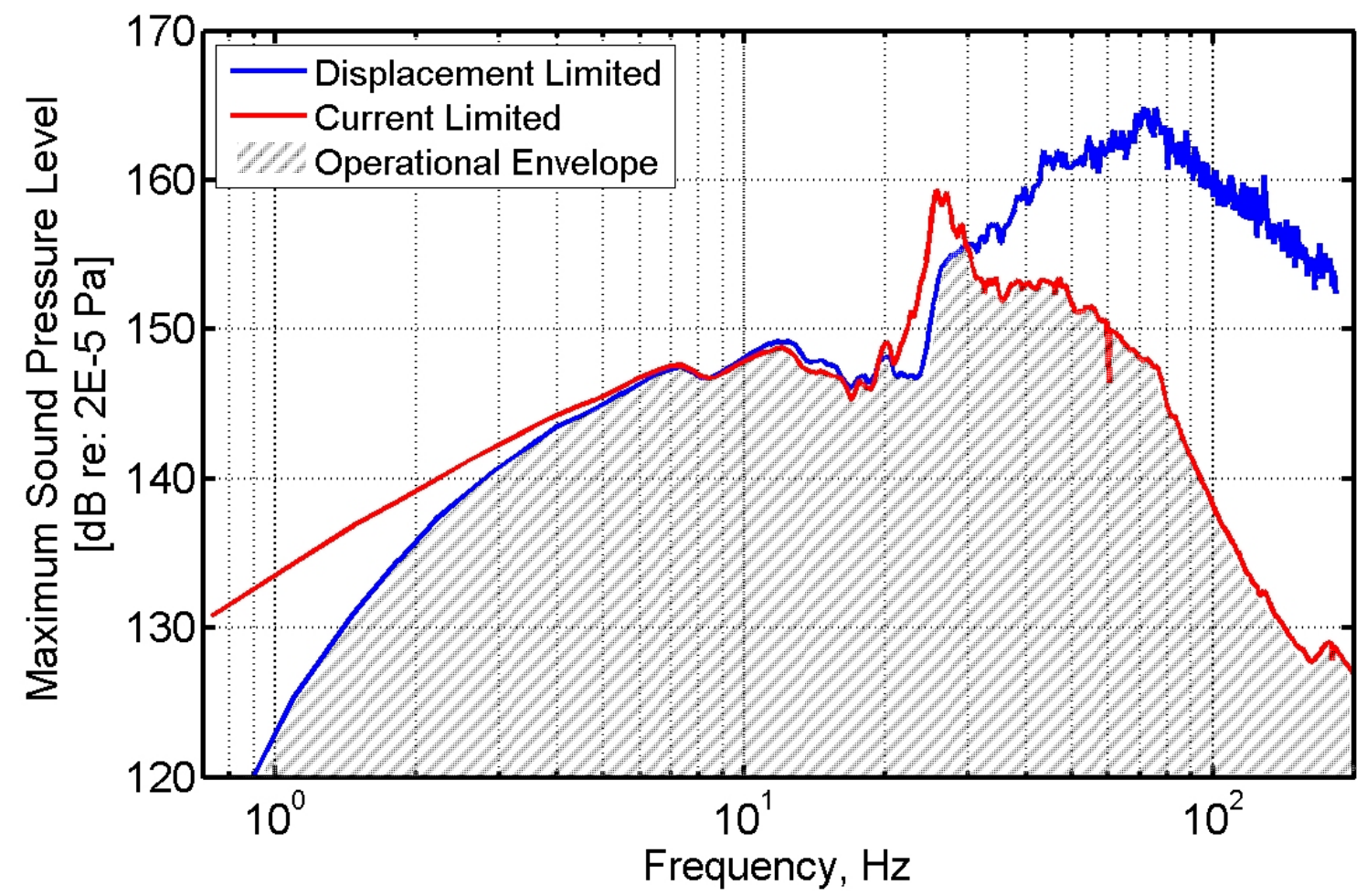

Fig. 13 - Maximum sound pressure level that can be produced at the exterior of the IER walls.

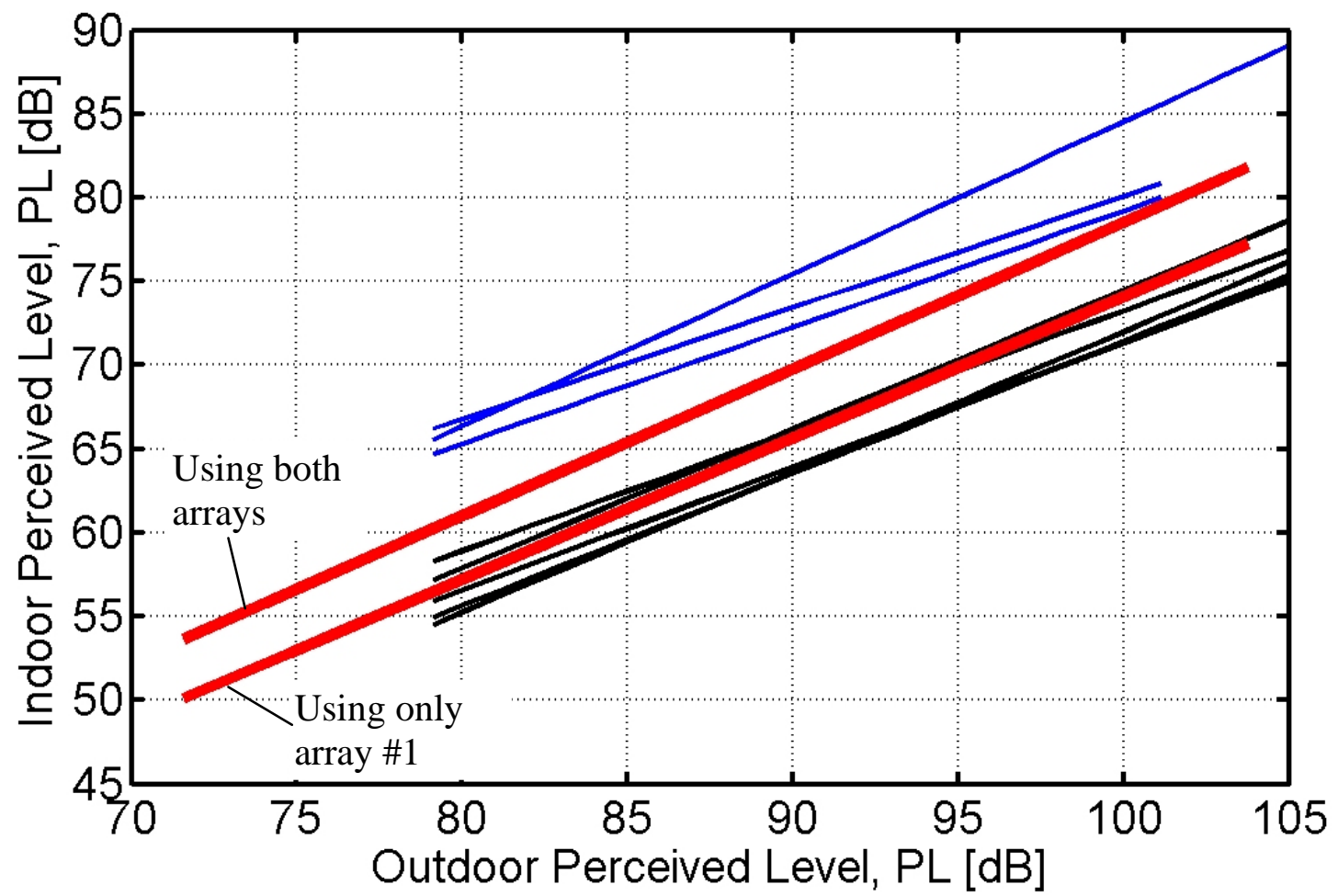

Fig. 14 - Interior versus exterior loudness; "- "IER with room configuration A (see Table 1) for two different array excitations, "- five different receiver locations in two rooms on the shadow side of a house $e^{5}, "-"$ three different locations in two rooms on the incident side of a house when the house was exposed to sonic booms from an F-18 aircraft. 
Boom playback through arrays 1 and 2
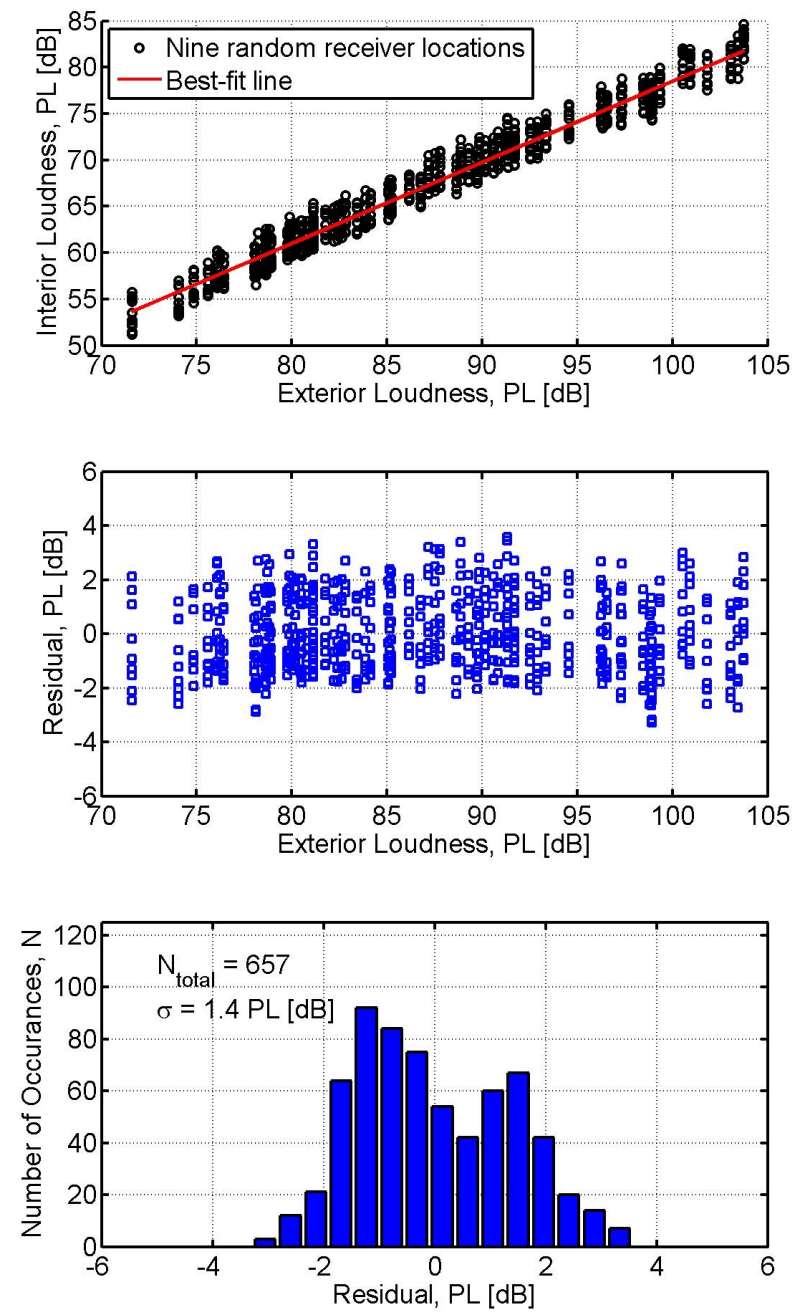

Boom playback through array 1 only
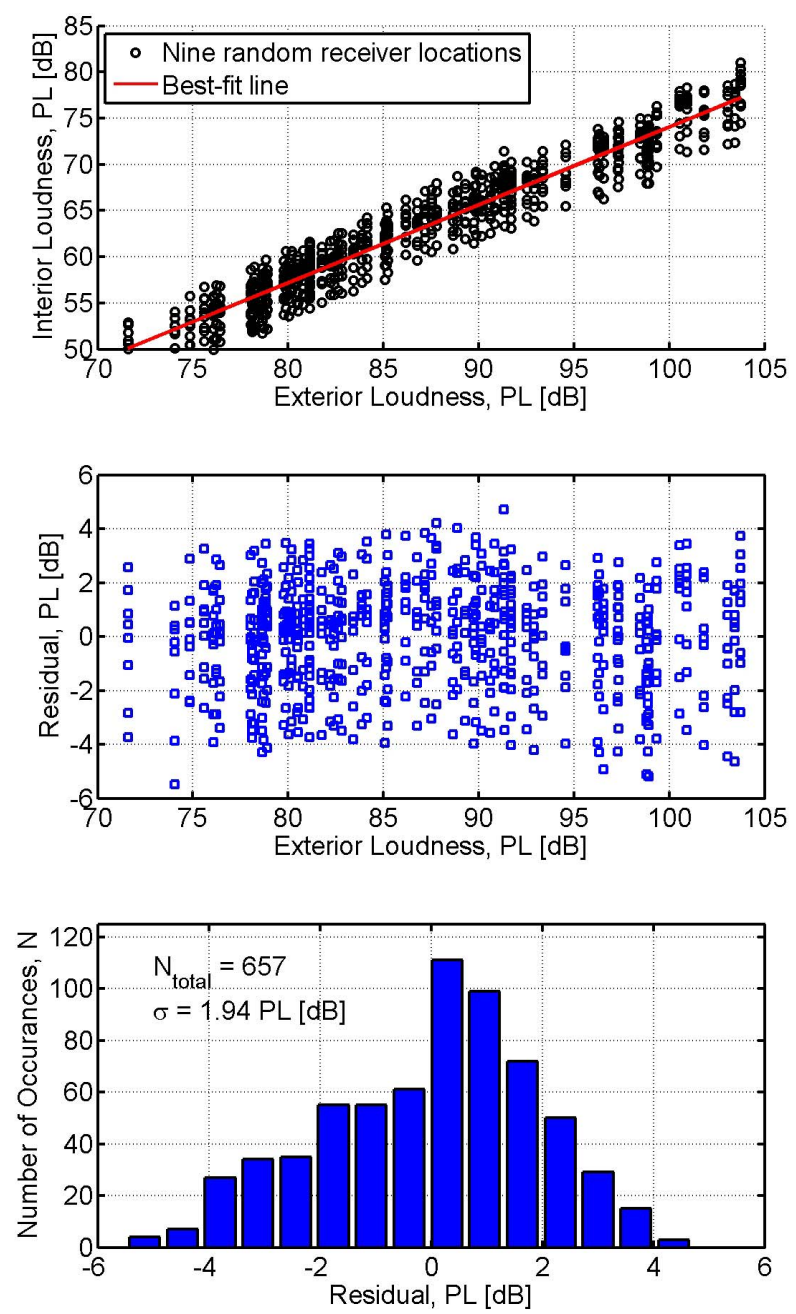

Fig. 15 - Interior loudness observed at nine randomly selected interior receiver locations for playback of seventy three different sonic booms for two different array configurations. Top row: individual PL values and the linear regression line, middle row: residuals versus exterior loudness, and bottom row: distribution and standard deviation of the residuals.

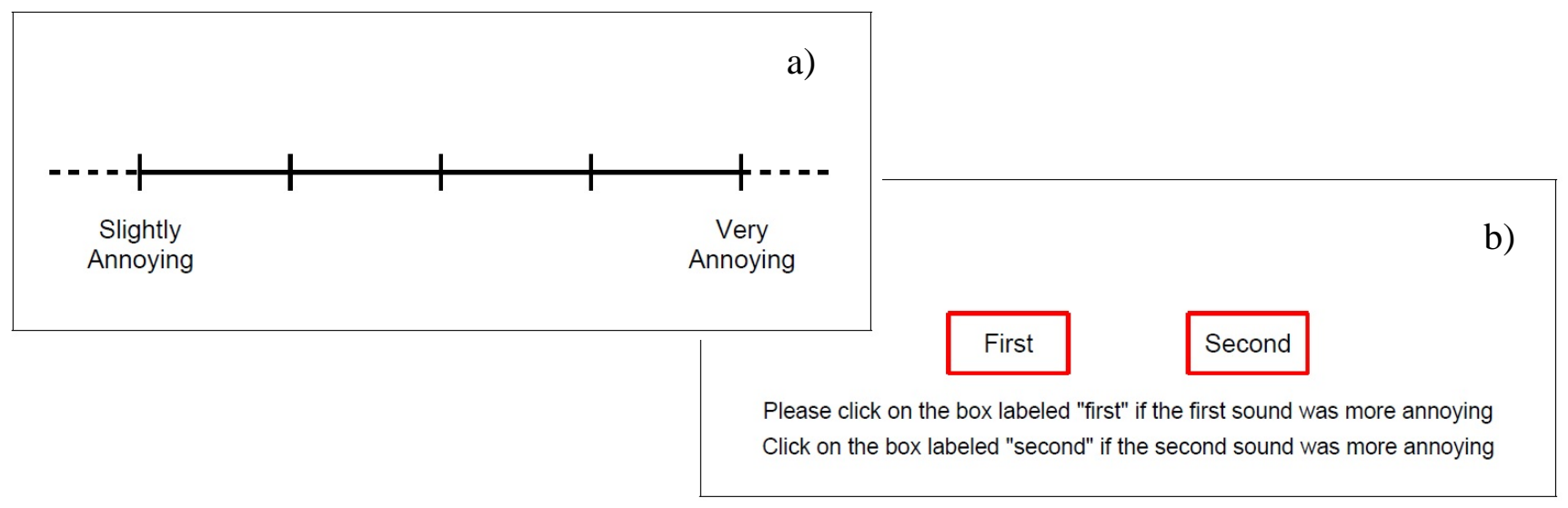

Fig. 16 - Typical judgment images presented to subjects on the remote computers during a) a category line scaling test and b) a paired comparison test. 